\title{
Cross-Over Assessment of the AmbuAuraGain, LMA Supreme New Cuff and Intersurgical I-Gel in Fresh Cadavers
}

\author{
Ana M. Lopez ${ }^{1}$, Xavier Sala-Blanch ${ }^{2}$, Ricard Valero ${ }^{3}$, Alberto Prats ${ }^{4}$ \\ ${ }^{1}$ Department of Anaesthesiology, Hospital Clínic de Barcelona, Barcelona, Spain \\ ${ }^{2}$ Orthopaedic Anaesthesia Section, Department of Anaesthesiology, Hospital Clínic de Barcelona, \\ Barcelona, Spain \\ ${ }^{3}$ Neuroanaesthesia Section, Department of Anaesthesiology, Hospital Clínic de Barcelona, Barcelona, Spain \\ ${ }^{4}$ Laboratory of Surgical Neuroanantomy (LSNA), Human Anatomy and Embryology Unit, Faculty of Medicine, \\ Universitat de Barcelona, Barcelona, Spain \\ Email: analopez@clinic.ub.es, xsala@clinic.ub.es, rvalero@clinic.ub.es, aprats@ub.edu
}

Received 19 October 2014; revised 28 November 2014; accepted 23 December 2014

Copyright (C) 2014 by authors and Scientific Research Publishing Inc.

This work is licensed under the Creative Commons Attribution International License (CC BY).

http://creativecommons.org/licenses/by/4.0/

(c) (i) Open Access

\begin{abstract}
Background: The AmbuAuraGain is a new single-use supraglottic airway device with gastric channel designed to facilitate intubation. The aim of the study was to assess the anatomic position and the performance of the AuraGain in fresh cadavers compared to that of the Intersurgical i-gel and LMA Supreme New Cuff. Methods: The 3 devices were inserted in random order in 7 fresh cadavers without difficult airway criteria. The assessed items were: Insertion time, number of attempts and ease of insertion, airway seal pressure, ease of gastric tube insertion, endoscopic view of vocal cords, efficacy of guided tracheal intubation through the AuraGain and i-gel, and anatomic fit by lateral X-ray and neck dissections. Results: All devices were successfully inserted within 3 attempts, except for one case of the LMA Supreme. Adjusting manoeuvres were often required to accomplish correct insertion. A 16 G gastric tube was easily advanced through all AuraGain and LMA Supreme devices. Fiberoptic tracheal intubation was effectively achieved through all AuraGain and i-gel devices in less than 60 s. Lateral X-ray and neck dissections confirmed optimal alignment of all devices with the respiratory and digestive tracts. Conclusions: Insertion of the new AmbuAuraGain required adjusting manoeuvres in some cases, as observed with the other two devices, and achieved similar airway seal pressures. Passage of a large bore gastric tube was as fast as with the LMA Supreme and ease of guided intubation was similar to that of the i-gel.
\end{abstract}

\section{Keywords}

Supraglottic Airway Device, Cadaver Study, Fibreoptic Intubation

How to cite this paper: Lopez, A.M., Sala-Blanch, X., Valero, R. and Prats, A. (2014) Cross-Over Assessment of the AmbuAuraGain, LMA Supreme New Cuff and Intersurgical I-Gel in Fresh Cadavers. Open Journal of Anesthesiology, 4, 332-339. http://dx.doi.org/10.4236/ojanes.2014.412047 


\section{Introduction}

The use of second generation supraglottic airway devices (SAD) is increasing exponentially since their introduction [1]. The combination of two main features, the improved airway seal and the ability to evacuate gastric contents, has contributed to extend the use of a SAD to a wider range of patients and indications. The LMA Supreme $^{\mathrm{TM}}$ (LMA Laryngeal Mask Co. Ltd, Le Rocher, Victoria, Mahe Seychelles) and i-gel ${ }^{\mathrm{TM}}$ (Intersurgical Ltd., Wokingham, England) were the first single use, second generation SAD introduced in 2007 and are widely used in clinical practice.

The LMA Supreme has been proven to be a safe and effective ventilation device with gastric channel that enables an easy passage of a large bore gastric tube [2]. The airway conduit does not permit a direct passage of a tracheal tube (TT) but fibreoptic guided intubation is possible with the aid of tube introducers. In 2013, the design and the materials of the LMA Supreme were modified [3]. The LMA Supreme New Cuff (LMA Teleflex INC, Limerick, Pennsylvania, USA) does not have the reinforced cuff on the posterior aspect of the gastric channel exit resulting in a less consistent tip (Figure 1 ).

The i-gel (Figure 1) has been reported to provide an effective airway with low morbidity rate [4] and to facilitate easy-guided tracheal intubation [5]. Although it features an oesophageal vent, the maximum size of the gastric tube that can be used is restricted to $14 \mathrm{G}$ for i-gel sizes 4 and 5, and $12 \mathrm{G}$ for size 3 .

Both devices have been extensively evaluated and compared pair-wise in different clinical scenarios. A recent meta-analysis of randomly controlled studies [6] concluded that the LMA Supreme and i-gel were similar in terms of insertion success rate, airway seal pressure and fibreoptic view.

The new AmbuAuraGain ${ }^{\mathrm{TM}}$ (Ambu A/S, Ballerup, Denmark) is a modification of the previous Ambu Aura$\mathrm{i}^{\mathrm{TM}}$, a SGA designed to facilitate guided tracheal intubation, which incorporates a gastric channel wide enough to accommodate a $16 \mathrm{G}$ gastric tube in all adult sizes and a slightly modified cuff to improve the seal pressure (Figure 1).

In this pre-clinical evaluation in fresh cadavers we assessed the performance and anatomic position of the AmbuAuraGain compared to that of the LMA Supreme and the i-gel. The aim of the study was to assess the ease of insertion, seal pressure, efficacy of gastric access, anatomical fit and capability of working as a conduit for direct optical intubation and extubation.

\section{Methods}

The procedures were performed at the Laboratory of Surgical Neuroanatomy (LSNA) within the Human Anatomy and Embryology Unit, Universit at de Barcelona. Approval from the Department Board was obtained. Permission to use the bodies for research or education was obtained upon donation. The study was performed in February 2014.

Eight fresh cadavers, 4 male and 4 female, without apparent difficult airway criteria were studied. The 3 devices were inserted in random order in all cadavers following a computer-generated sequence.

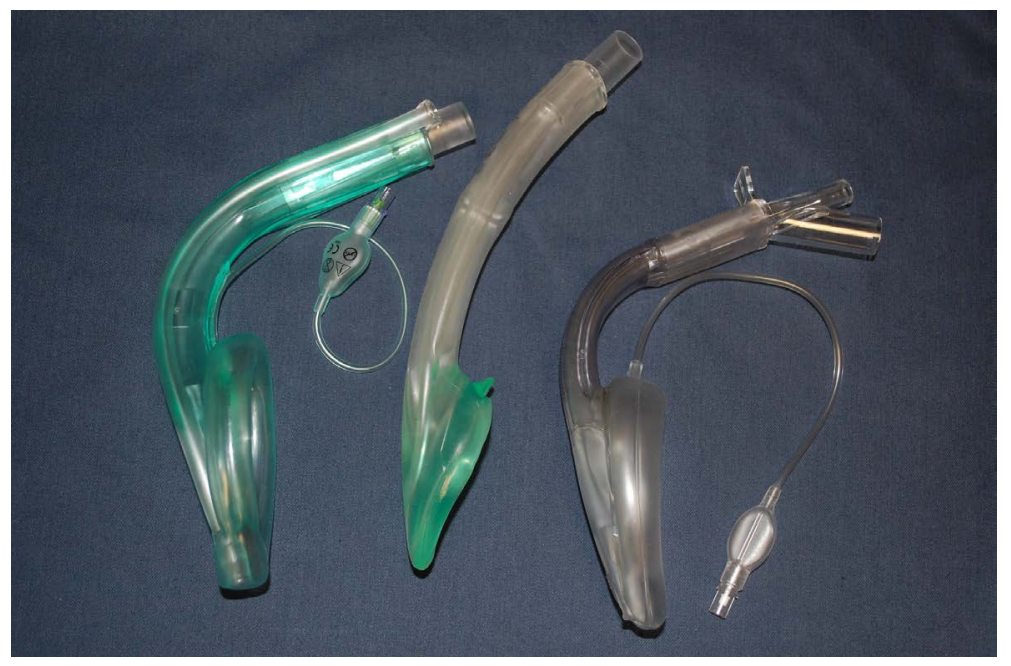

Figure 1. Lateral view of the AmbuAuraGain, i-gel and LMA Supreme. 
Prior to device insertion, we evaluated the following parameters of the airway: mouth opening and dentition, mandibular subluxation, neck mobility, thyro-mental distance and neck circumference. The head was placed in neutral position elevated $8 \mathrm{~cm}$ on a firm support. A direct laryngoscopy was performed to obtain the percentage of glottis opening [7].

Size of SGA was selected according to the manufacturer's recommendations based on the estimated weight and height of the bodies. All SGA were lubricated with water-based gel. The cuffs of the AuraGain and the LMA Supreme were fully deflated prior to insertion. All devices were inserted by the same an aesthetist (AML).

The standard recommended insertion technique, pushing the device along the hard palate was followed for the first attempt. In case of resistance to insertion, adjusting manoeuvres were applied as paramedian approach by lateral rotation of the cuff, jaw lift, head and neck extension, redirection of the tip with the finger. For the second attempt, a gastric tube was advanced through the gastric channel $10 \mathrm{~cm}$ beyond the tip and the device was blindly inserted [8]. If the second attempt failed, a preloaded gastric tube or tube introducer was advanced into the oesophagus under direct laringoscopy to guide the insertion of the device [9]. In case of failure at third attempt, the assessment was stopped. The cuffs of the AmbuAuraGain and the LMA Supreme were inflated to a $60 \mathrm{~cm} \mathrm{H}_{2} \mathrm{O}$ pressure with a manometer (Ambu Cuff Pressure Gauge, Ambu A/S, Ballerup, Denmark). Qualitative assessment of each insertion attempt was as follows: Easy, if no resistance was felt along the mouth and pharynx; acceptable, if moderate resistance was encountered requiring mask rotation or additional jaw lift; and difficult if the resistance persisted, requiring manipulation of the tip or the tongue with the contralateral finger. Insertion time was defined as from when the tip of the SGA was placed into the mouth until it was completely introduced. We did not include the time for inflation of the AuraGain and LMA Supreme. If more than one attempt was needed, the sum of all insertion times was recorded.

To confirm the correct position of the devices (as ventilation was not reliable in all cadavers), endoscopic view of vocal cords was performed using an AmbuaScope 2 (5.4 mm diameter, Ambu A/S, Ballerup, Denmark). The position of the SGA was scored based on the highest percentage of glottic opening (POGO) visualized. For LMA Supreme size 3, a lateral X-ray was performed due to inability to introduce the scope into the airway tube.

Efficacy of gastric access was tested by recording the time to insert the maximum recommended size of a gastric tube, Pennine Levin, $60 \mathrm{~cm}$ beyond the proximal end. For LMA Supreme and AmbuAuraGain, a 16 G gastric tube was used for all sizes. For i-gel, 14 and 12 G gastric tubes were inserted through sizes 4 or 5 , and 3 respectively. Number of insertion attempts, time and qualitative assessment as easy, acceptable or difficult, were also registered.

Seal pressure was measured after confirming the correct position of the device. A continuous air flow of $6 \mathrm{~L}$ per min was connected to the airway tube until the equilibrium pressure was reached. The mean of three consecutive measures was accepted for each device.

Guided intubation with the a Scope 2 was performed through the AuraGain and i-gel using the maximum recommended tube sizes: 8, 7.5 and $6.5 \mathrm{~mm}$ ID for AuraGain sizes 5, 4 and 3 respectively and 8, 7 and 6 mm ID for i-gel sizes 5, 4 and 3 respectively. The aScope, loaded with the TT (Mallindckrodt, Covidien, Mansfield, MA, USA) was advanced through the airway tube until the carina was seen. The TT tube was then railroaded over the aScope and the position was verified. Intubation time was measured from when the tip of the aScope entered into the SGA connector until endoscopic confirmation of correct ETT placement. Number of intubation attempts required, ease of passage of the aScope, ease of passage of the tube and overall ease of intubation.

A lateral X-ray of the neck was obtained for each device with the gastric tube in place to compare their positions in relation to the anatomic structures of the respiratory and digestive tracts. Specifically, we evaluated the relation between the proximal border of the cuff to the hyoid bone and the base of the tongue, the position of the tip in relation to the upper oesophagus and the alignment of the airway tube with the glottic inlet.

At the end of the investigation, the necks of two cadavers were dissected to assess the position of the cuff and anatomic fit of the three devices.

As this study was intended as pre-clinical assessment, the sample size was not powered to compare the three devices and therefore, no statistical analysis was performed.

\section{Results}

One of the male cadavers was excluded due to difficult laryngoscopy (POGO 0\%) and the impossibility to insert all three devices within 3 attempts due to local anatomical alteration. None of the 7 remaining cadavers had difficult airway criteria or POGO $<30 \%$ on direct laryngoscopic view. 
All devices were successfully placed and rated as easy or acceptable to insert, except one size 3 LMA Supreme that failed at third attempt because of repeated malposition of the tip into the airway (Table 1, Figure 2).

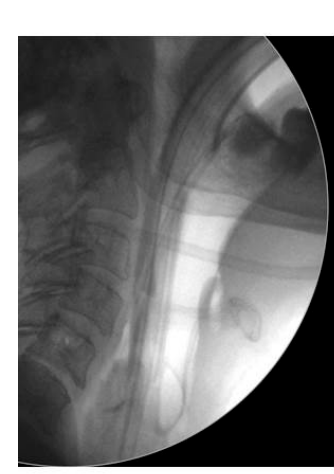

First attempt

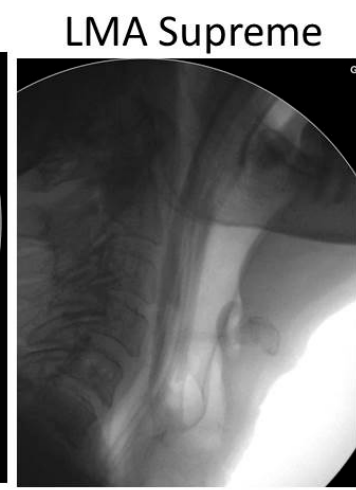

Second attempt

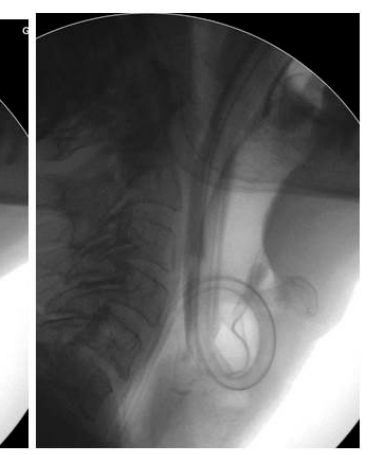

Third attempt

Figure 2. Malposition of the LMA Supreme New Cuff in one female cadaver. First attempt: recommended insertion technique. The tip of the cuff and the gastric went inside the trachea. Second attempt: The gastric tube was advanced first into the esophagus to guide the tip of the mask. The image shows the tip caught at the level of arythenoids. Third attempt: the gastric tube was first advanced into the esophagus under direct vision to guide the insertion, however the tip of the mask entered again the glottis pushing the gastric tube inside.

Table 1. Details of the supraglottic devices tested and their performance.

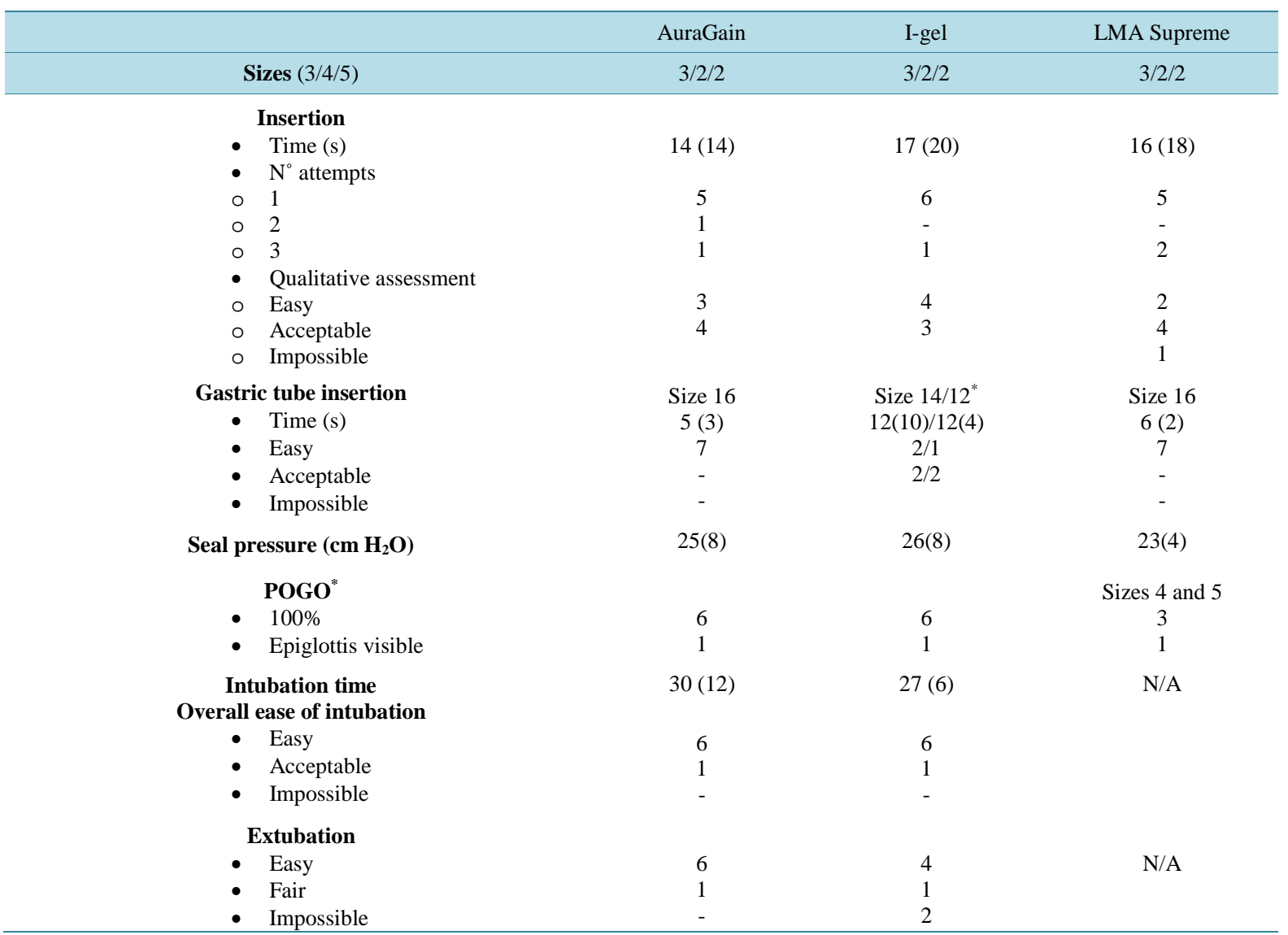

Data expressed as number of cases or mean (standard deviation). POGO: Percentage of glottis opening; *: only in size 3 i-gel. 
Adjusting manoeuvres, as lateral or paramedian approach, jaw lift, head/neck mobilization, were often required to overcome some degree of resistance to insertion with all three devices. However, the difficulty was encountered at different levels depending on the SGA. The resistance in advancing the AuraGain was found when the tip reached of the palate-pharyngeal curve. The passage of the i-gel was restricted at the level of oral cavity if the tongue was caught inside the bowl. The LMA Supreme was easily advanced into the hypopharinx but the tip impacted often with the arytenoids, causing misplacement into the glottis in some cases. Reinsertion was successful in all but one cadaver, even using the described guided technique (Figure 2).

Gastric tube (16 G) insertion was fast and rated as easy for all AuraGain and LMA supreme devices, while the insertion of a gastric tube $14 \mathrm{G}$ or $12 \mathrm{G}$ through the corresponding sizes of i-gel was rated as acceptable in three cases (Table 1).

Seal pressure was above $20 \mathrm{~cm} \mathrm{H}_{2} \mathrm{O}$ for all three devices (Table 1). The passage of the aScope was not possible through the airway connector of size 3 LMA Supreme. Fibreoptic view revealed an optimal alignment of the airway tube and vocal cords in all cases with the AuraGain and i-gel. In two of these patients, the tip of the epiglottis was visible inside the conduit (Table 1) although it did not obstruct the view or the passage of the aScope and TT, which were considered easy or acceptable in all cases. Although the manufacturer of i-gel recommends smaller TT sizes, the intubation was attempted also with a 6.5 and $7 \mathrm{~mm}$ ID TT sizes through the i-gel sizes 3 and 4 respectively, with the same results as with the AuraGain.

Lateral X-ray of neck confirmed the correct final position of the three devices in relation to the airway and the placement gastric tube into the esophagus (Figure 3).

The anterior displacement of the hyoid bone and laryngeal structures was more evident with the i-gel when the volume needed to achieve an intracuff pressure of $60 \mathrm{~cm} \mathrm{H}_{2} \mathrm{O}$ was low with the AuraGain and LMA Supreme (Figure 3). Interestingly, the proximal edge of the cuff of the size 5 AuraGain appeared slightly wrinkled in two cadavers in comparison to the cuff of size 5 LMA Supreme (Figure 4).

Dissection of two necks showed the correct position of the bowl around the glottis inlet with all three brands of SGAs assessed (Figure 5).

\section{Discussion}

This pre-clinical evaluation in fresh cadavers shows that the performance of the AuraGain is in line with that of the i-gel or LMA Supreme with respect to ease of insertion, anatomic position, efficacy of gastric access and capability to serve as an intubating conduit.

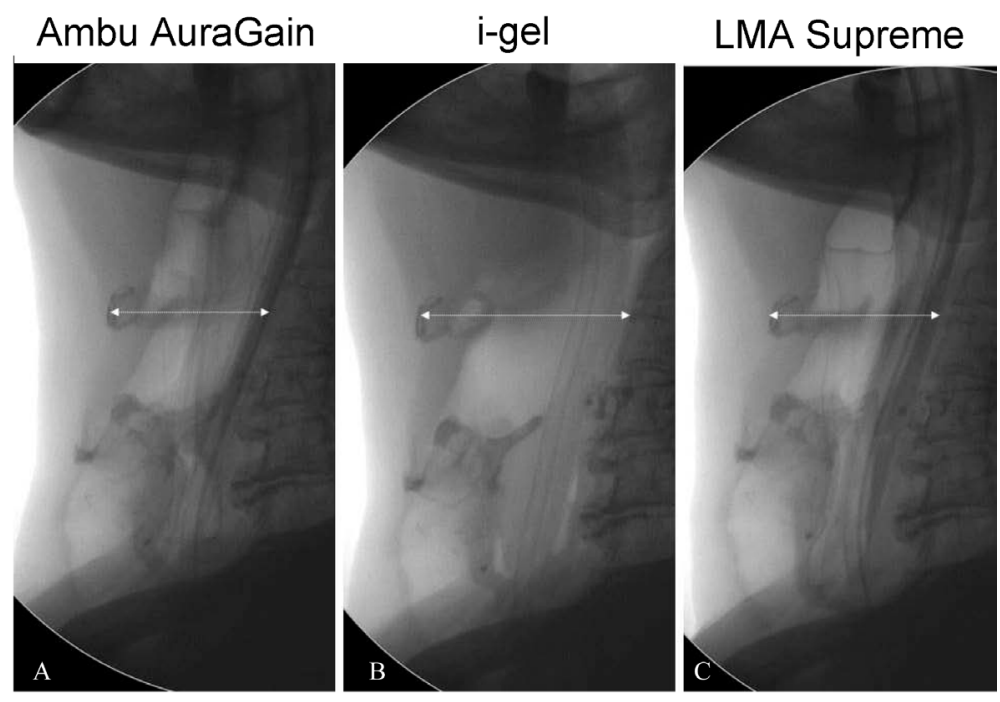

Figure 3. Lateral X-Ray images of the three devices (size 4) in the same cadaver. (A) AmbuAuraGain; (B) Intersurgical i-gel; (C) LMA Supreme. The arrows show the distance from the spine to the hyoid bone. The cuffs of the AuraGain and the LMA Supreme were both inflated to $60 \mathrm{~cm} \mathrm{H}_{2} \mathrm{O}$ causing less anterior displacement of the perilaryngeal structures. 

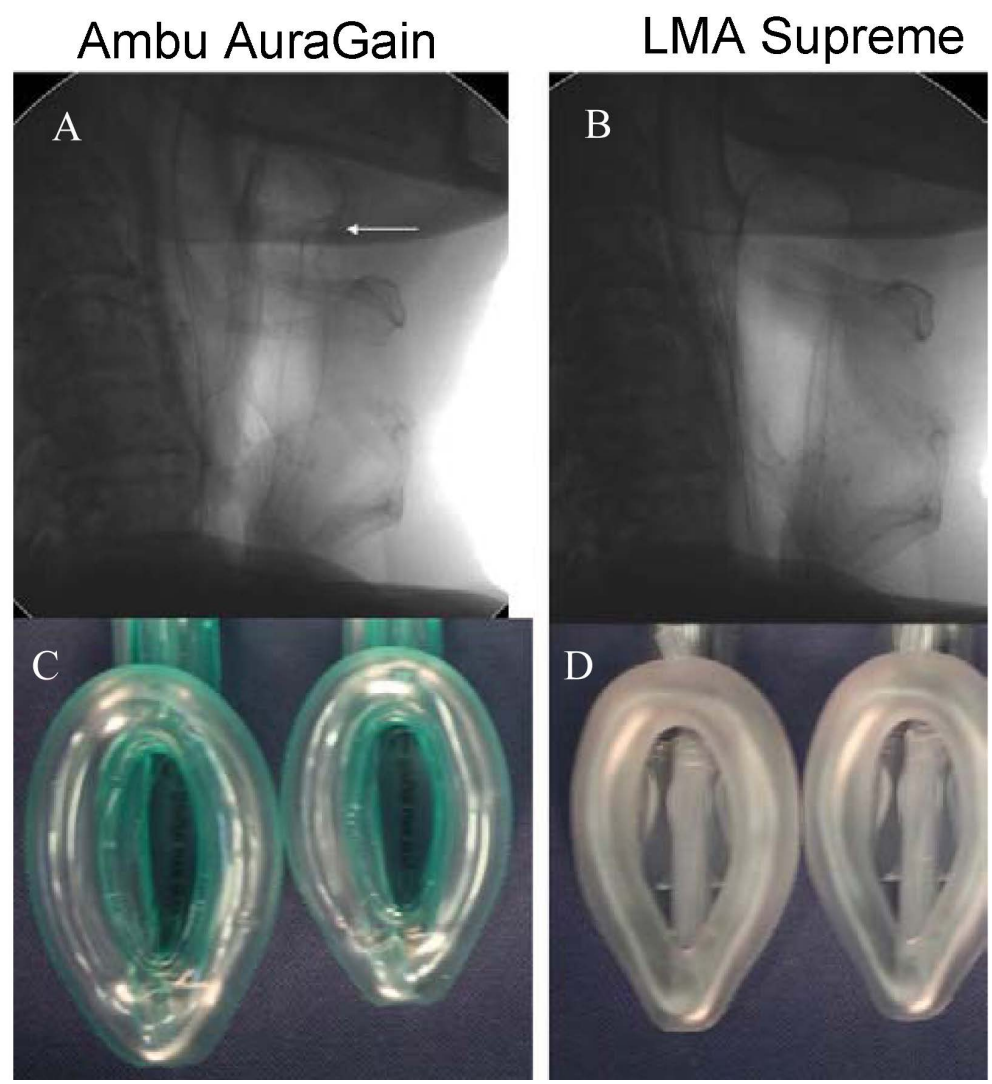

Figure 4. Lateral X-Ray of the Size 5 AmbuAuraGain (A) and LMA Supreme (B). A: The arrow shows the proximal end of the cuff of the AuraGain that appears slightly wrinkled in comparison to the same size of the LMA Supreme New Cuff. Size 5 and 4 of the AuraGain (C) and LMA Supreme (D). Cuff dimensions of the AuraGain size 5 are larger than that of size 4. The LMA Supreme that has the same cuff for sizes 4 and 5 .
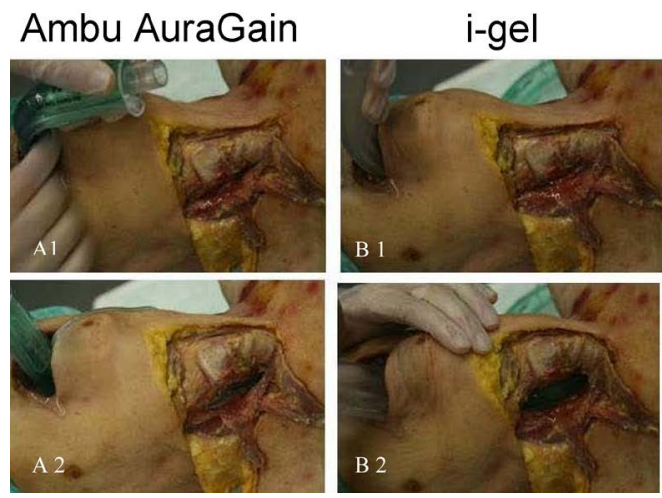

\section{LMA Supreme}

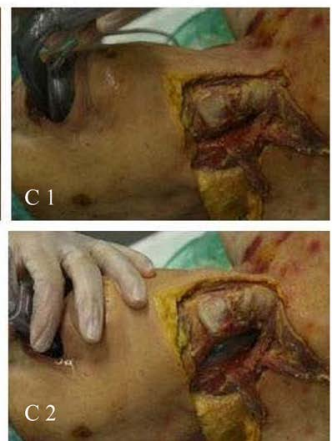

Figure 5. Dissection of the lateral neck showing the laryngeal structures with the AmbuAuraGain (A); I-gel (B) and LMA Supreme (C) before insertion (1) and the final position of the cuff in relation to the airway structures (2).

Differences in design and materials determine the differences found in the course of insertion in our study. For instance, the firm tip of the AuraGain does not bend easily toward the hypopharinx after hitting the posterior pharyngeal wall, that was solved by lateral rotation inside the mouth [4]. The solid cuff of the i-gel found some resistance to pass beneath the tongue along the midline in some cadavers, requiring the same adjusting manoeuvre in some cases. And finally, the irregular tip of the LMA Supreme New Cuff seems to make it more prone to 
collide with the arytenoids and enter into the glottis. The potential impact of these modifications has not been assessed in clinical studies yet.

Despite that the gastric channel exists at a $20^{\circ}$ angle from the midline of the AuraGain, the passage of a large bore gastric tube along the esophagus was as easy and fast as with the LMA Supreme in all cadavers and all masks sizes tested. This suggests that, once in place, the tip of the gastric channel is properly aligned with the oesophagus allowing effective vent of eventually regurgitated gastric contents. In contrast, the gastric channel of the i-gel accommodates a maximum $14 \mathrm{G}$ tube through sizes 4 and 5 , and $12 \mathrm{G}$ through the size 3 . The difficulty in advancing the gastric tube found in some cases was related to the narrower and less gliding surface of the i-gel channel. Significant differences on ease of insertion of the gastric tube between LMA Supreme and i-gel have been previously reported [6] [10].

The seal pressure obtained with the three devices were slightly lower than those reported for the i-gel and LMA-Supreme in clinical studies [6], although higher than that commonly achieved with first generation devices. The better seal of the second generation devices is obtained by an improved wedge-shape of the cuff. The antero-posterior diameter of the proximal bowl is larger, allowing for a tighter fit to the peri-laryngeal structures (Figure 1). This shape can be obtained either by increasing the diameter of the proximal cuff (LMA Supreme), the diameter of the tube (AuraGain), or both (i-gel). Our results suggest that the three designs are equally effective to seal the airway, although the pressure against the surrounding structures and the consequent distortion of the anatomy may be different [11].

Capability of acting as an intubating conduit was similar for the AuraGain and the i-gel in terms of fibreoptic view, passage of the aScope and ease of TT insertion. Although the manufacturer of the i-gel recommends smaller TTs sizes, intubations were successful in all cases using the maximum allowed tube sizes for both devices. This should be taken cautiously, as external diameters of the TTs may vary with the manufactures. The presence of the epiglottis did not restrict fibreoptic guided intubation, however, it may prevent the blind introduction of the TT, resulting in a low success rate [5] [12]. Therefore, flexible scopes should be recommended to guide intubation though these devices.

The X-ray images showed the correct alignment with the airway and digestive tract for all three devices after successful insertion. The proximal border of the cuff in two Size 5 AuraGain devices appeared slightly wrinkled compared to the same size of LMA Supreme, suggesting the size of mask was not the appropriate in spite of the estimated height and weight of the cadavers. Cuff dimensions of the AuraGain size 5 are larger than that of the LMA Supreme, which has the same cuff for sizes 4 and 5 (Figure 4).

The main limitation of the study is that the lack of tone and elasticity of tissues in the cadaver limit the extrapolation of data to clinical practice. Although, previous studies have shown that ease of insertion, pharyngeal compliance and seal pressure observed in fresh cadavers and anesthetized paralyzed patients were similar using different laryngeal masks [13] [14], we found a less easy insertion and lower seal pressure than in clinical routine practice for the i-gel and LMA supreme.

In conclusion, the new AuraGain achieved a seal pressure in the range of that obtained with LMA Supreme and i-gel, allowed a fast gastric access with a large bore gastric tube and facilitated tracheal intubation. The combination of these three features in a single device offers a promising alternative for airway management in challenging patients and advanced indications. The satisfactory performance of the AuraGain needs to be confirmed in clinical evaluation.

\section{Acknowledgements}

The authors thank Eduard Latorre for his enthusiastic and helpful contribution to obtain the RX images.

\section{References}

[1] Hernandez, M.R., Klock Jr., P.A. and Ovassapian, A. (2012) Evolution of the Extraglottic Airway: A Review of Its History, Applications, and Practical tips for Success. Anesthesia and .Analgesia, 114, 349-368. http://dx.doi.org/10.1213/ANE.0b013e31823b6748

[2] Wong, D.T., Yang, J.J. and Jagannathan, N. (2012) Brief Review: The LMA Supreme ${ }^{\text {TM }}$ Supraglottic Airway. Canadian Journal of Anesthesia, 59, 483-493. http://dx.doi.org/10.1007/s12630-012-9673-0

[3] Lopez, A.M., Valero, R., Lopez, S., Zaballos, M. and Garcia, M.L. (2013) LMA Supreme: New Design or a Pig in a Poke? Revista Española de Anestesiología y Reanimación, 60, 597-598. http://dx.doi.org/10.1016/j.redar.2013.06.017 
[4] Theiler, L., Gutzmann, M., Kleine-Brueggeney, M., Urwyler, N., Kaempfen, B. and Greif, R. (2012) I-Gel ${ }^{\mathrm{TM}}$ Supraglottic Airway in Clinical Practice: A Prospective Observational Multicentre Study. British Journal of Anaesthesia, 109, 990-995. http://dx.doi.org/10.1093/bja/aes309

[5] Kleine-Brueggeney, M., Theiler, L., Urwyler, N., Vogt, A. and Greif, R. (2011) Randomized Trial Comparing the $\mathrm{I}-G e l^{\mathrm{TM}}$ and Magill Tracheal Tube with the Single-Use ILMA ${ }^{\mathrm{TM}}$ and ILMA ${ }^{\mathrm{TM}}$ Tracheal Tube for Fibreoptic-Guided Intubation in Anaesthetized Patients with a Predicted Difficult Airway. British Journal of Anaesthesia, 107, 251-257. http://dx.doi.org/10.1093/bja/aer103

[6] Chen, X., Jiao, J., Cong, X., Liu, L. and Wu, X. (2013) A Comparison of the Performance of the I-Gel vs. the LMA-S ${ }^{\mathrm{TM}}$ during Anesthesia: A Meta-Analysis of Randomized Controlled Trials. PLoS One, 8, e71910. http://dx.doi.org/10.1371/journal.pone.0071910

[7] Levitan, R.M., Ochroch, E.A., Kush, S., Shofer, F.S. and Hollander, J.E. (1998) Assessment of Airway Visualization: Validation of the Percentage of Glottic Opening (POGO) Scale. Academic Emergency Medicine, 5, 919-923. http://dx.doi.org/10.1111/j.1553-2712.1998.tb02823.x

[8] Garcia-Aguado, R., Vinoles, J., Brimacombe, J., Vivo, M., Lopez-Estudillo, R. and Ayala, G. (2006) Suction Catheter Guided Insertion of the ProSeal Laryngeal Mask Airway Is Superior to the Digital Technique. Canadian Journal of Anesthesia, 53, 398-403. http://dx.doi.org/10.1007/BF03022507

[9] Brimacombe, J., Keller, C. and Judd, D.V. (2004) Gum Elastic Bougie-Guided Insertion of the ProSeal Laryngeal Mask Airway Is Superior to the Digital and Introducer Tool Techniques. Anesthesiology, 100, 25-29. http://dx.doi.org/10.1097/00000542-200401000-00008

[10] Teoh, W.H., Lee, K.M., Suhitharan, T., Yahaya Z., Teo, M.M. and Sia, A.T. (2010) Comparison of the LMA Supreme vs the I-Gel in Paralysed Patients Undergoing Gynaecological Laparoscopic Surgery with Controlled Ventilation. Anaesthesia, 65, 1173-1179. http://dx.doi.org/10.1111/j.1365-2044.2010.06534.X

[11] Russo, S.G., Cremer, S., Eich, C., Jipp, M., Cohnen, J., Strack, M., Quintel, M. and Mohr, A. (2012) Magnetic Resonance Imaging Study of the in Vivo Position of the Extraglottic Airway Devices I-Gel ${ }^{\mathrm{TM}}$ and LMA-Supreme in Anaesthetized Human Volunteers. British Journal of Anaesthesia, 109, 996-1004. http://dx.doi.org/10.1093/bja/aes314

[12] Halwagi, A.E., Massicotte, N., Lallo, A., Gauthier, A., Bordreault, D., Ruel, M. and Girard, F. (2012) Tracheal Intubation through the I-Gel Supraglottic Airway versus the LMA Fastrach: A Randomized Controlled Trial. Anesthesia \& Analgesia, 114, 172-178. http://dx.doi.org/10.1213/ANE.0b013e318236f438

[13] Keller, C., Brimacombe, J.R., Radler, C., Puhringer, F. and Brimacombe, N.S. (2000) The Intubating Laryngeal Mask Airway: Effect of Handle Elevation on Efficacy of Seal, Fibreoptic Position, Blind Intubation and Airway Protection. Anaesthesia and Intensive Care, 28, 414-419.

[14] Brimacombe, J. and Keller, C. (1999) The Laryngeal Mask Airway in Fresh Cadavers versus Paralysed Anaesthetized Patients: Ease of Insertion, Airway Sealing Pressure, Intracuff Pressures and Anatomic Position. European Journal of Anaesthesiology, 16, 699-701. http://dx.doi.org/10.1046/j.1365-2346.1999.00568.x 
Scientific Research Publishing (SCIRP) is one of the largest Open Access journal publishers. It is currently publishing more than 200 open access, online, peer-reviewed journals covering a wide range of academic disciplines. SCIRP serves the worldwide academic communities and contributes to the progress and application of science with its publication.

Other selected journals from SCIRP are listed as below. Submit your manuscript to us via either submit@scirp.org or Online Submission Portal.
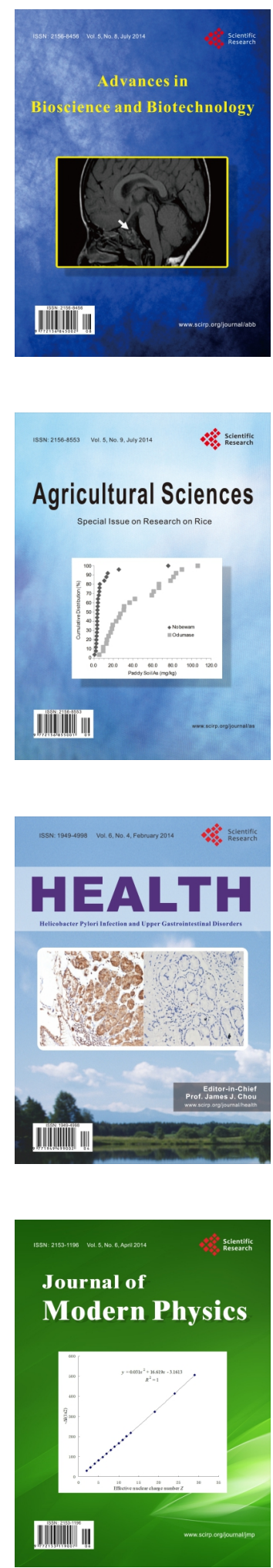
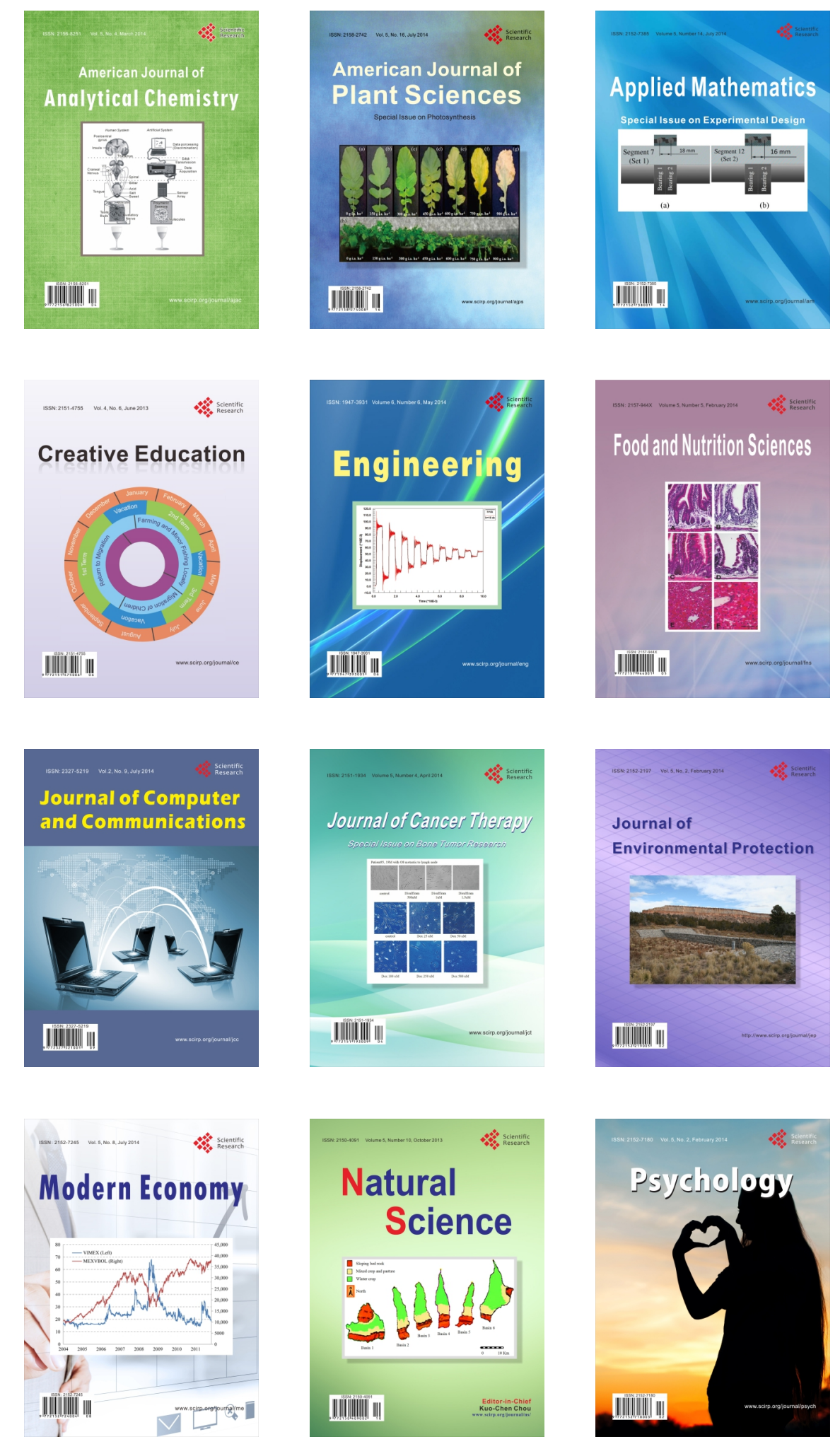